\title{
ENTWICKLUNG UND UNTERSUCHUNG EINER LEICHTEN, FUNKTIONSIN- TEGRIERTEN KAROSSERIE IN METALL-SANDWICH-BAUWEISE
}

Michael Kriescher, Marc Hampel, Thomas Grünheid, Simon Brückmann,

(DLR Institut für Fahrzeugkonzepte)

Katja Oswald

(Dow Automotive)

\section{ABstract}

Am Institut für Fahrzeugkonzepte des Deutschen Zentrums für Luft- und Raumfahrt (DLR) wird im Rahmen des Verbundforschungsprojektes Next Generation Car (NGC) ein sehr leichtes Fahrzeugkonzept der L7e-Klasse als Forschungsdemonstrator mit einem Karosseriegewicht von nur $90 \mathrm{~kg}$ entwickelt, das gleichzeitig sehr gute Crasheigenschaften aufweist. Das Strukturkonzept beinhaltet die konsequente Anwendung von Hybrid-Werkstoffen in einer Sandwichbauweise, so dass sich eine leichte Struktur ergibt, die aus vergleichsweise wenigen, einfach geformten Bauteilen besteht. Diese Struktur wird durch FE-Simulationen sowie durch statische und dynamische Versuche für verschiedene Crashlastfälle untersucht.

Die hohen Anforderungen an Gewicht und Crashsicherheit erfordern außerdem ein leichtes, auf die Crashszenarien abgestimmtes Fahrwerkskonzept, das ebenfalls simulativ und experimentell untersucht wird. Die Ergebnisse dieser Untersuchungen werden hier vorgestellt.

\section{PRojeKTZIELE}

Die Realisierung batterieelektrischer Fahrzeuge mit mittlerer bis hoher Reichweite ist bislang noch schwierig, vor allem aufgrund der geringen spezifischen Speicherkapazität aktueller Batteriesysteme. Das Forschungsprojekt Next Generation Car (NGC) adressiert unter anderem diese Problematik. Im Teilprojekt Safe Light Regional Vehicle (SLRV) wird ein sehr leichtes, aerodynamisch günstiges Fahrzeug der L7e-Klasse als Forschungsdemonstrator entwickelt, das geringe Fahrwiderstände und damit einen geringen Energiebedarf aufweist. Da bis zu 2/3 des Energiebedarfs gewichtsabhängig sind [Friedrich \& Hülsebusch, 2009], ist ein geringes Fahrzeuggewicht ein wesentlicher Faktor für die Reduzierung des Energiebedarfs.

Um eine Reichweite von 400 km zu erzielen, wird das Fahrzeug mit einem Brennstoffzellen-Batterie-Hybrid-Antrieb ausgelegt. Die Höchstgeschwindigkeit beträgt $120 \mathrm{~km} / \mathrm{h}$.

Ein wesentliches Projektziel ist trotz des sehr geringen Karosseriegewichtes von nur 90 kg, eine Crashsicherheit zu erreichen, die weitgehend dem Stand der Technik in der M1-Klasse entspricht. Dabei sollen die Kosten für die Herstellung der Karosserie durch die Verwendung weniger, relativ einfach geformter Bauteile aus konventionellen Werkstoffen gering gehalten werden.

\section{KAROSSERIEKONZEPT}

Um die Projektziele zu erreichen, wird eine sehr leichte Karosseriestruktur entwickelt, die nahezu vollständig aus SandwichElementen besteht. Die Verwendung teurer Werkstoffe wie CFK wird möglichst vermieden. Stattdessen werden SandwichStrukturen mit metallischen Decklagen für die wesentlichen Strukturbauteile verwendet. Solche Sandwich-Bauteile wurden bislang selten im Automobilbau eingesetzt, da sie normalerweise nur für kastenförmige Strukturen mit geraden Wänden angewandt werden [Davies \& Bryant, 2011].

Das Konzept des SLRV verwendet hingegen im Bereich der Bodenwanne dreidimensional geformte Sandwichbauteile, die im oberen Teil mit einer mit Schaum gefüllte Ringstruktur schließen, so dass eine metallische Monocoque-Struktur entsteht. Im Bereich von Vorder- und Hinterwagen werden dagegen kastenförmige Strukturen aus geraden kostengünstigeren Platten verwendet (Abbildung 1).

Die Karosserie ist so gestaltet, dass ihre Herstellung nur wenige Werkzeuge und damit vergleichsweise geringe Investitionskosten erfordert, was eine Produktion schon bei geringen bis mittleren Stückzahlen profitabel macht. 
Der elektrische Antriebsstrang bietet dabei eine hohe Gestaltungsfreiheit, die für die Erstellung einer sehr leichten, einfachen Struktur genutzt wird.

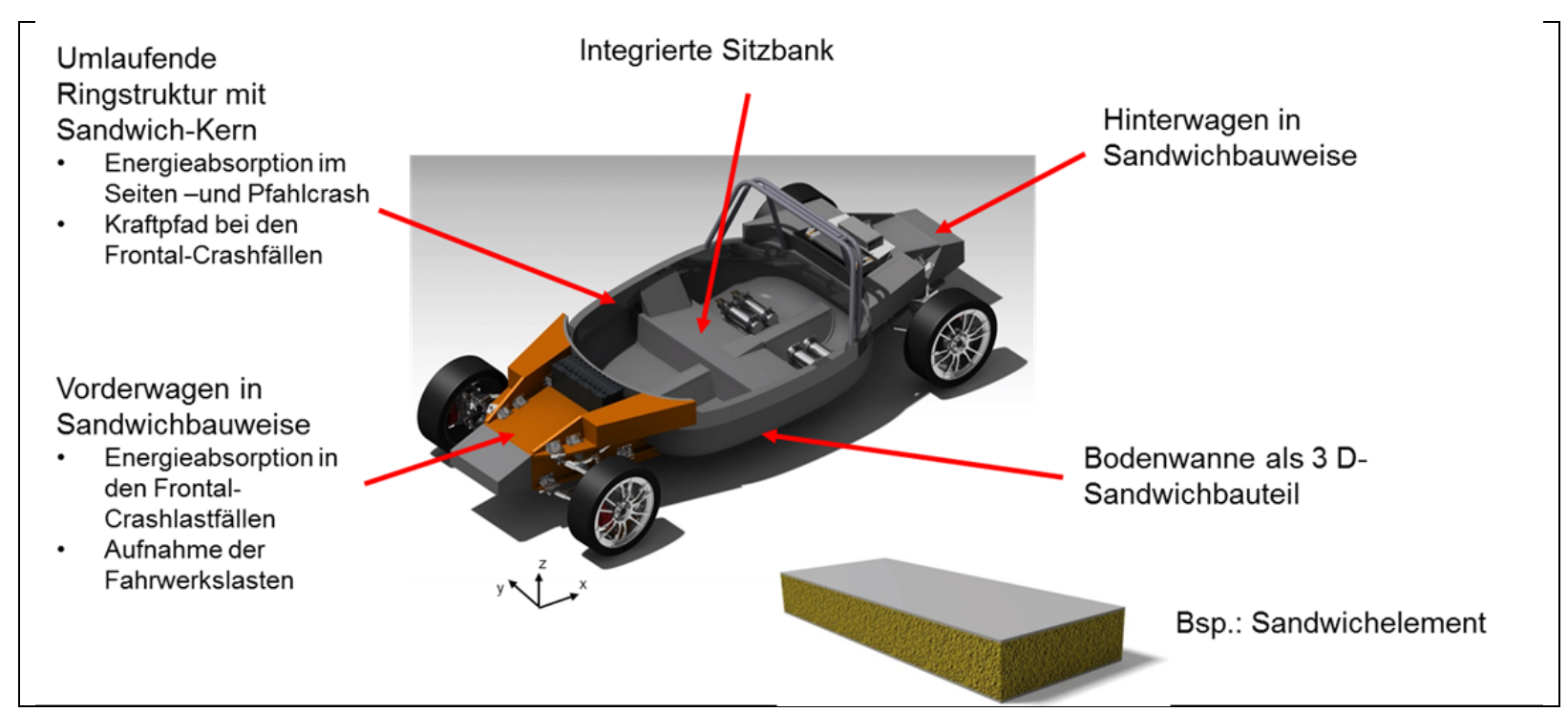

Abbildung 1: Karosserie in Sandwichbauweise, rechts Sandwichplatte aus metallischen Deckschichten mit Kern aus Kunststoff-Schaum

\section{ERGEBNISSE DER CRASHSIMULATIONEN}

Um wesentliche Crasheigenschaften der Karosserie zu validieren, wurden verschiedene Crashlastfälle simuliert:

- $\quad$ US NCAP Frontalaufprall, $56 \mathrm{~km} / \mathrm{h}$

- $\quad$ Euro NCAP ODB-Aufprall (Offset Deformable Barrier Crash), 64 km/h

- $\quad$ Euro NCAP Pfahlaufprall, $29 \mathrm{~km} / \mathrm{h}$

- $\quad$ Euro NCAP Seitenaufprall, $950 \mathrm{~kg}, 50 \mathrm{~km} / \mathrm{h}$

Dabei wurde zunächst ein vereinfachtes Karosseriemodell verwendet, bei dem noch nicht alle konstruktiven Details integriert sind. Dieses Berechnungsmodell wird im Laufe des Projektes immer weiter detailliert. Um die Crashsicherheit der Karosserie zu bewerten, werden die auftretenden Intrusionen mit den Intrusionen an einer in konventioneller Metall-Schalenbauweise aufgebauten Referenz-Karosserie verglichen, die den Stand der Technik repräsentiert.

Im Gegensatz zu einer Fahrgastzelle aus hohlen Strukturen kann die metallische Sandwichstruktur des SLRV eine gewisse Deformation ertragen, ohne dass es zu einem Beulen oder Knicken kommt. Dies ist auf die Stützwirkung der Sandwichkerne zurückzuführen, die ein frühes Beulen der metallischen Außenhaut verhindern. Verglichen mit einer Karosserie nach dem Stand der Technik sind die Intrusionen der SLRV-Karosserie bei Seiten- und Pfahlaufprall geringer (Tabelle 1). Lediglich beim Euro NCAP ODB-Aufprall treten etwas höhere Intrusionen auf.

Tabelle 1: Maximale Intrusion der SLRV-Karosserie im Vergleich zu einer Referenzkarosserie nach dem Stand der Technik

\begin{tabular}{lll}
\hline & Max. Intrusion & Max. Intrusion \\
Lastfall & SLRV-Karosserie & Referenz-Karosserie \\
& {$[\mathrm{mm}]$} & {$[\mathrm{mm}]$} \\
\hline Euro NCAP ODB-Aufprall & 102 & 84 \\
Euro NCAP Seitenaufprall & 155 & 211 \\
Euro NCAP Pfahlaufprall & 206 & 381 \\
\hline
\end{tabular}


Insbesondere beim Pfahlaufprall zeigt die Simulation eine vergleichsweise geringe Intrusion, mit einem gleichmäßigen Beschleunigungsverlauf (Abbildung 2). Besonders bei diesem Lastfall kommt die Stützwirkung der Sandwichkerne zum Tragen. Im Falle der Ringstruktur verhindert eine Füllung mit PU-Schaum der Fa. Dow Automotive ein Kollabieren des Profils. Die Ringform sorgt für eine möglichst großflächige Verteilung der auftretenden Dehnungen über den Umfang des Rings (Abbildung 3 Mitte).

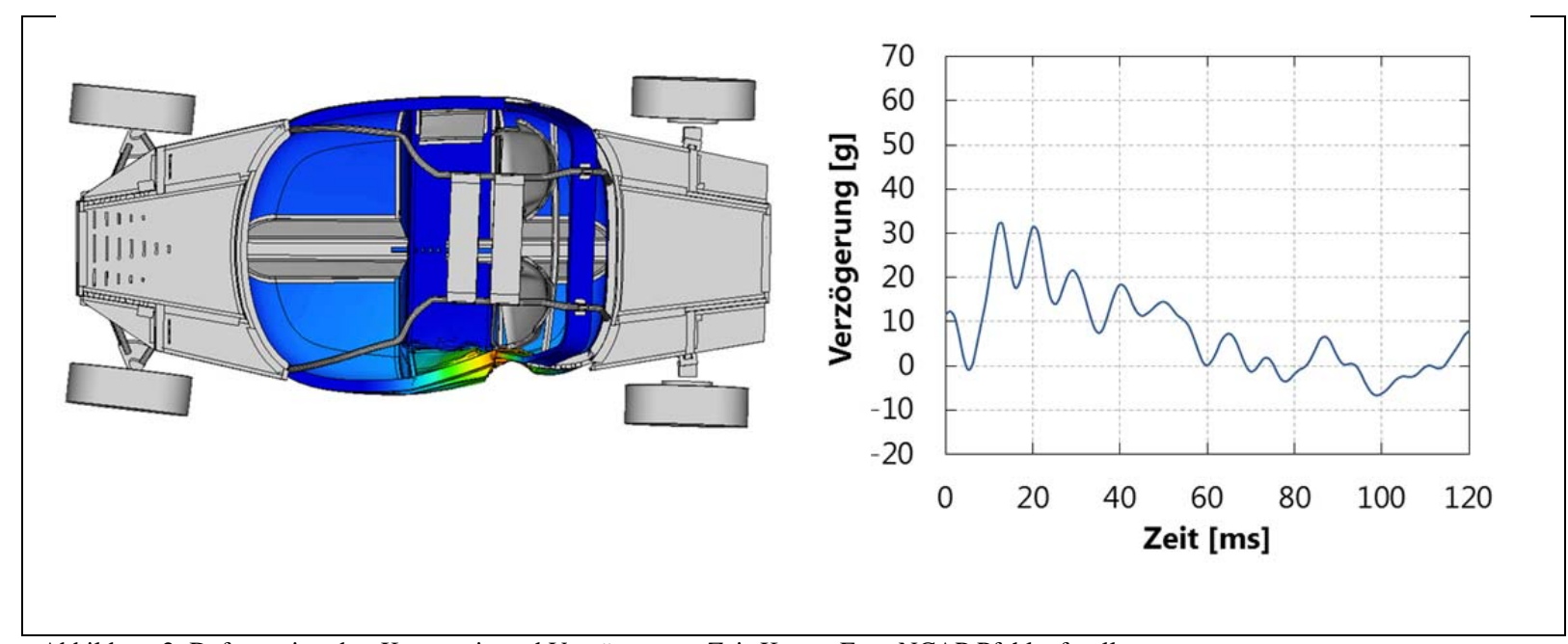

Abbildung 2: Deformation der Karosserie und Verzögerungs-Zeit-Kurve, Euro NCAP Pfahlaufprall

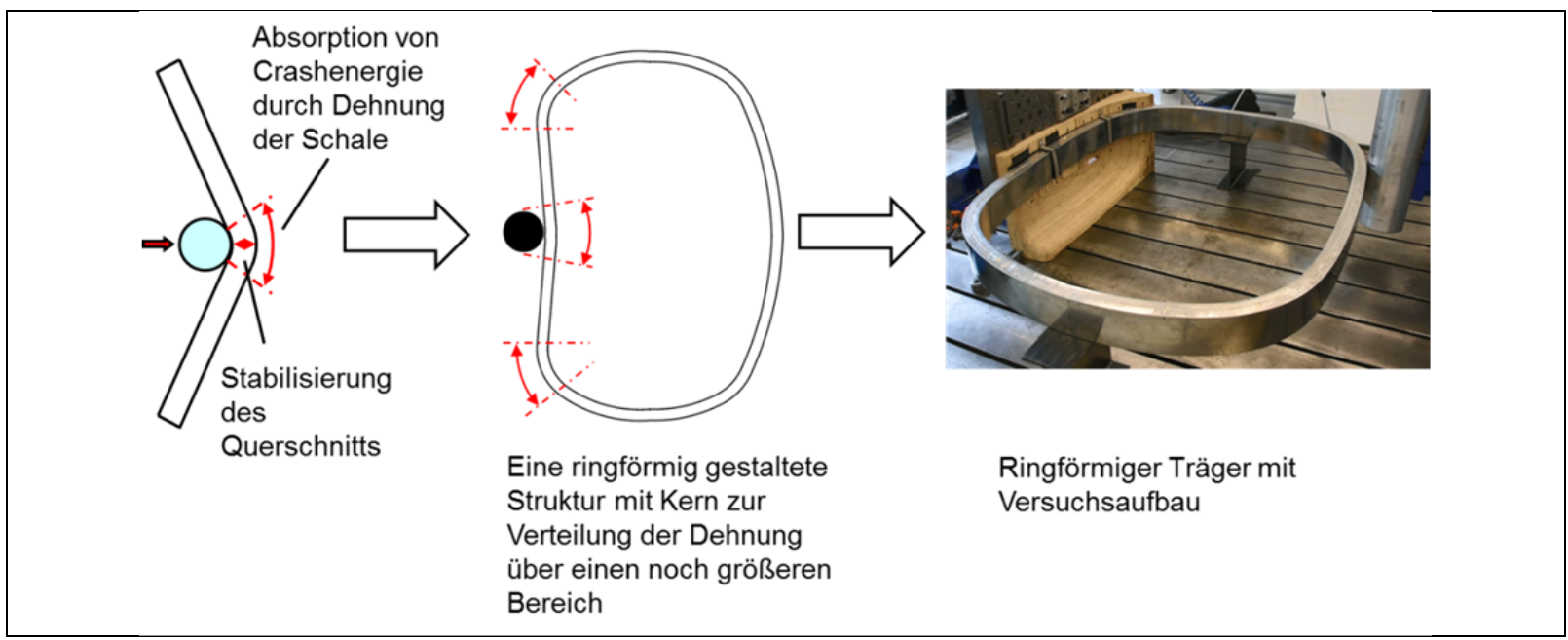

Abbildung 3: Wirkungsweise des ringförmigen Trägers und Versuchsaufbau

\section{VERSUCHSERGEBNISSE}

Eine Herausforderung bei der Crashsimulation von Sandwichstrukturen ist insbesondere die Modellierung des Versagensverhaltens der Kerne, die in diesem Fall aus Kunststoff-Schaum bestehen. Versuche an Proben und Bauteilen sind daher für die Beurteilung des Crashverhaltens, sowie für einen Abgleich von Simulation und Realität, notwendig.

\subsection{QUASISTATISCHE VERSUCHE AN RINGSTRUKTUREN}

Auf dem Hydraulischen Prüffeld des Institutes für Fahrzeugkonzepte wurden quasistatische Eindrückversuche an Ringstrukturen durchgeführt. Für den Versuch wurde ein zylindrischer Prüfkörper mit einem Durchmesser von 254 mm eingesetzt. Die Position wurde so gewählt, dass sie dem Aufprallpunkt eines Euro-NCAP Pfahlaufpralls entspricht. Die Prüfgeschwindigkeit lag bei $1,5 \mathrm{~mm} / \mathrm{s}$. Als Gegenlager wurde eine der Ringkontur angepasste Holzform verwendet, um eine möglichst gleichmäßige Krafteinleitung zu gewährleisten. 
Der Versuch ergibt eine gleichmäßige Kraft-Weg-Kurve mit einem Kraftniveau von ca. 18 kN (Abbildung 4). Aufgrund der ermittelten Kraft-Weg-Kurve ist eine ausreichende Energieabsorption des Bauteils im Crashfall zu erwarten.

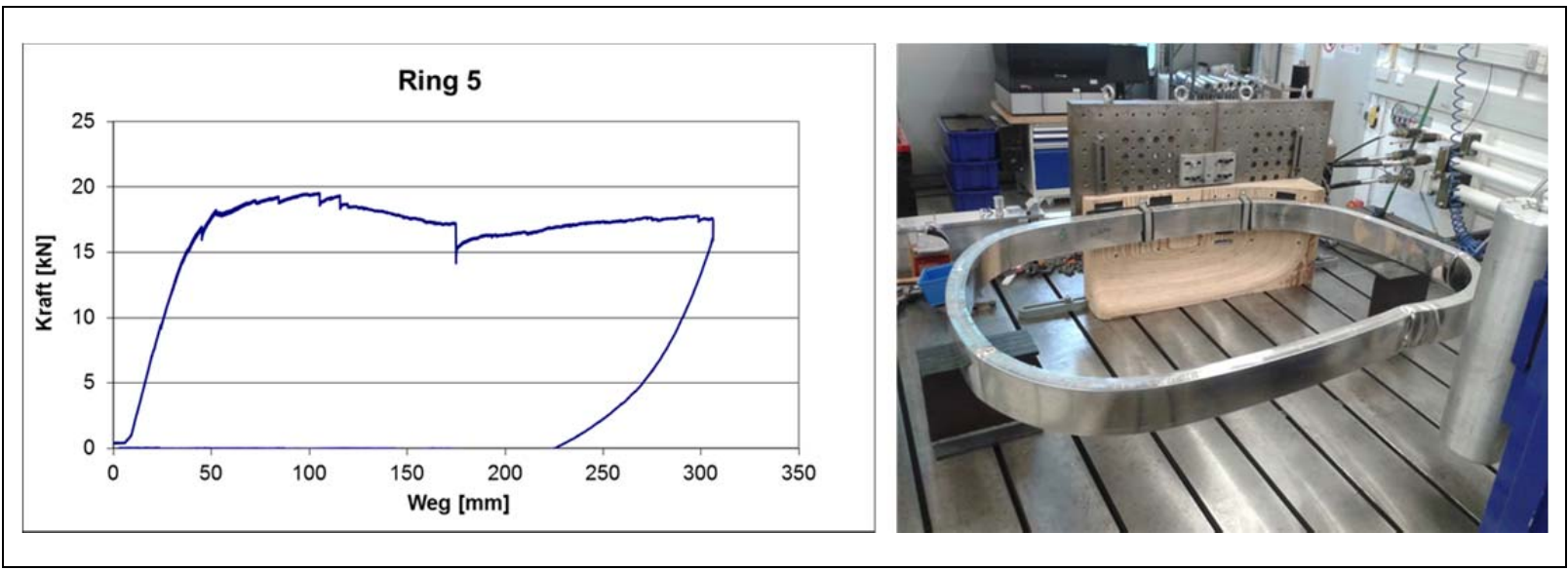

Abbildung 4: Quasistatischer Eindrücktest an einem mit PU-Schaum gefüllten Ring

\section{Auslegung Des FAHRWERKS}

Die Deformation der vorderen Radaufhängung ist wesentlich für das Verhalten der Karosserie bei den frontalen Crashlastfällen. Aufgrund der leichten Bauweise der Bodenwanne muss das Auftreffen des Vorderrades auf die Struktur verhindert werden. Die vordere Radaufhängung ist daher so gestaltet, dass das Rad an der Fahrgastzelle vorbeigeführt wird.

Dieses Fahrwerk wurde detailliert konstruiert und wird, sowohl durch Simulation als auch durch Crashversuche, validiert. Es handelt sich um ein Doppelquerlenker-Fahrwerk mit Dreiecks-Querlenkern, bei denen alle Elemente aus geraden Rohren bestehen, was ein sehr geringes Gewicht bei gleichzeitig hoher Steifigkeit garantiert (Abbildung 5).

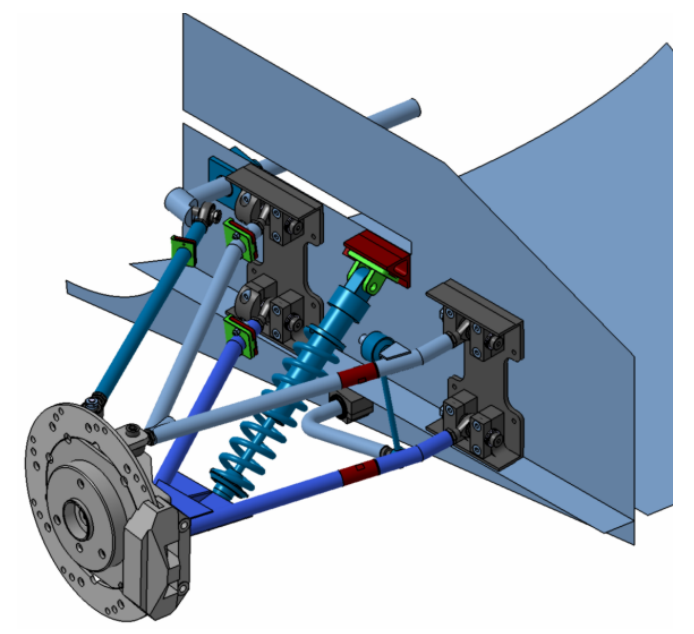

Abbildung 5: Konstruktion des vorderen Doppelquerlenker-Fahrwerks des SLRV

Um ein sicheres Vorbeileiten des Rades im Crashfall zu erzielen, werden mehrere Sollbruchstellen an den Fahrwerkselementen so ausgelegt, dass sie sukzessive während des Crashverlaufs versagen. Durch diese Abstimmung wird eine Drehbewegung erzeugt, die das Rad an der Fahrgastzelle vorbeiführt (Abbildung 6). Da die im Crashfall auftretenden Kräfte wesentlich höher sind als die Betriebslasten, kann ein sicherer Betrieb des Fahrwerks trotzdem gewährleistet werden. 
Der Vorgang des Ablösens kann in drei Phasen unterteilt werden: Als erstes reißen die Dehnschrauben in den Rohren der vorderen Querlenker bei Erreichen des oben genannten Kraftniveaus ab. Kurz darauf löst sich das Federbein, ebenfalls durch das Versagen einer Dehnschraube. Die Querlenker drehen sich anschließend um ihre hinteren Gelenke. Dadurch wird das Rad nach außen und an der Fahrgastzelle vorbei geführt. Die dritte Phase besteht im Ablösen der hinteren Querlenker-Rohre sowie der Spurstange. Dies wir durch Kraftbegrenzungselemente bewirkt, die gleichzeitig die Drehbewegung des Fahrwerks erzeugen. Somit wird erreicht, dass sich das Rad frei an der Karosserie vorbei bewegen kann.

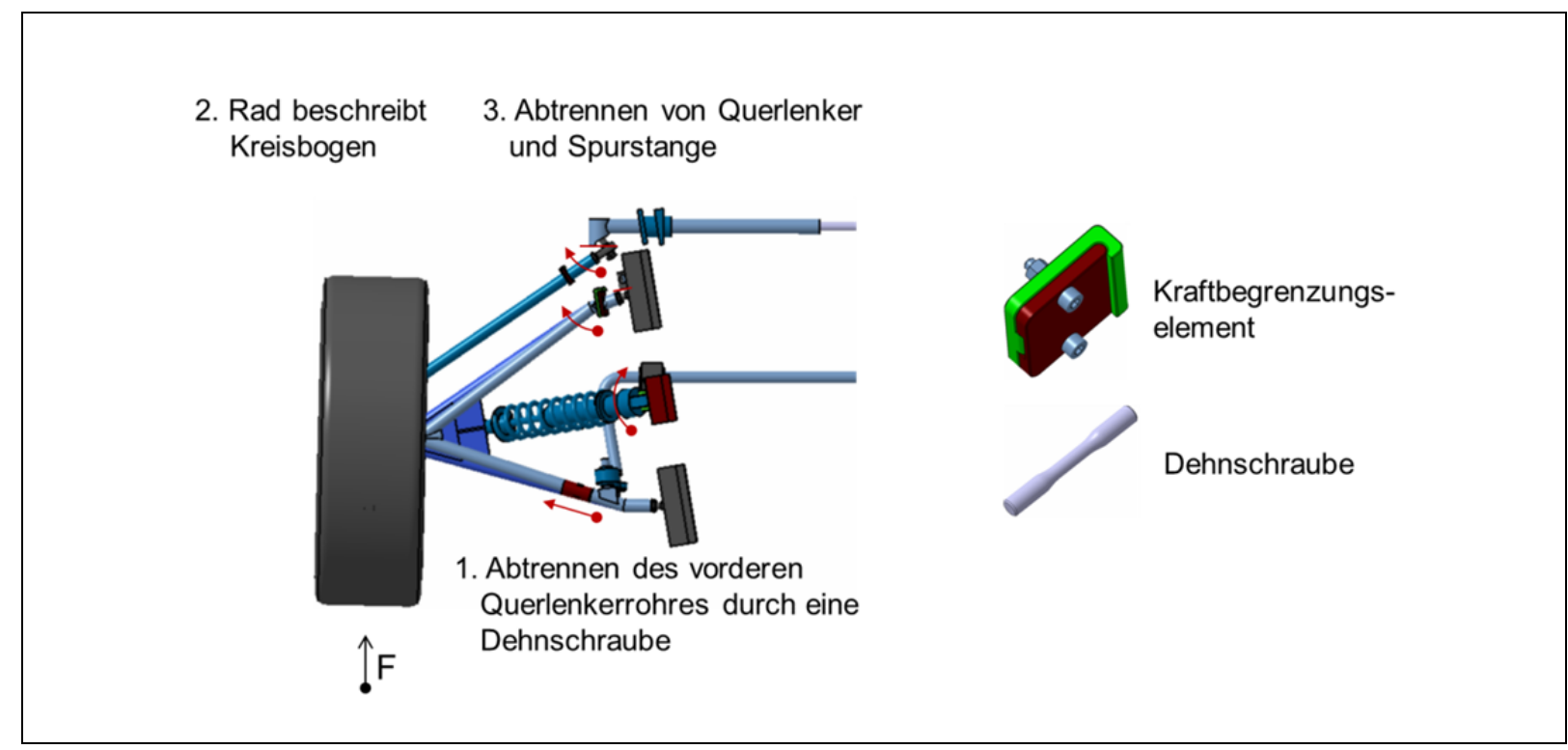

Abbildung 6: Kinematik des vorderen Doppelquerlenker-Fahrwerks des SLRV im Crashfall; rechts: im Fahrwerk integriertes Kraftbegrenzungselement und Dehnschraube

Die Simulation eines EURO NCAP ODB-Crashtests der SLRV-Karosserie mit angebrachtem Fahrwerk zeigt erfolgreich, dass durch die oben beschriebene Kinematik eine größere Beschädigung der Fahrgastzelle durch Rad und Fahrwerk vermieden werden kann.

\section{Versuche mit der Fahrwerksanbindung}

Ein wesentlicher Aspekt der Fahrwerksauslegung ist die Festigkeit der Anbindung an die Vorderwagenstruktur des Fahrzeugs. Um diese zu untersuchen wurde eine Teilstruktur eines Sandwich-Vorderwagens als Versuchsaufbau realisiert. Die Anbindung erfolgt durch großflächiges Verkleben von Verteilerplatten aus Aluminium (Abbildung 7). Entscheidend ist dabei die Krafteinleitung in die horizontalen Sandwichplatten des Vorderwagens. Diese wird durch zusätzliche Klebeflächen oben und unten, die in die Verteilerplatten integriert sind, realisiert.

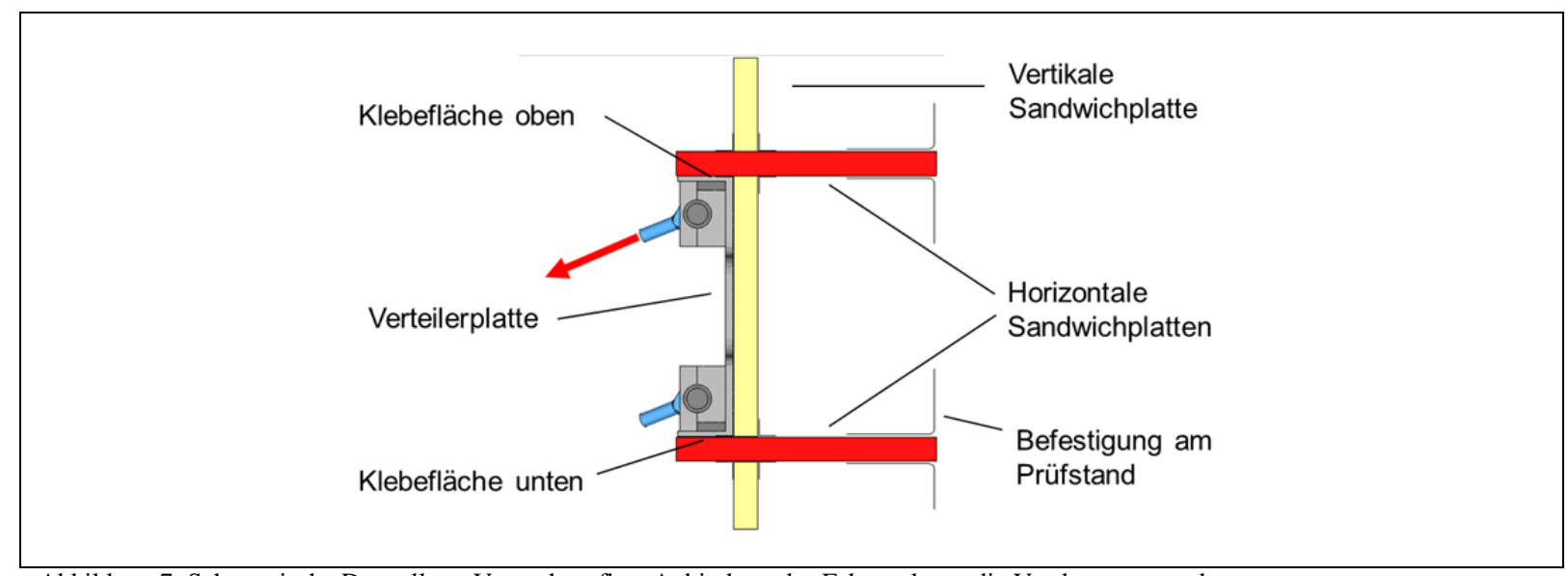

Abbildung 7: Schematische Darstellung Versuchsaufbau Anbindung des Fahrwerks an die Vorderwagenstruktur

Durch diesen Prüfaufbau lässt sich an einem Anbindungspunkt eine maximale Kraft von rund 23 kN übertragen (Abbildung 8), eine Vorschädigung des Bauteils ist bereits bei $21 \mathrm{kN}$ erkennbar. Die für das definierte Abtrennen des Querlenkers im Crashfall (Abbildung 6) notwendige Kraft lässt sich dadurch mit mehr als zweifacher Sicherheit übertragen. 

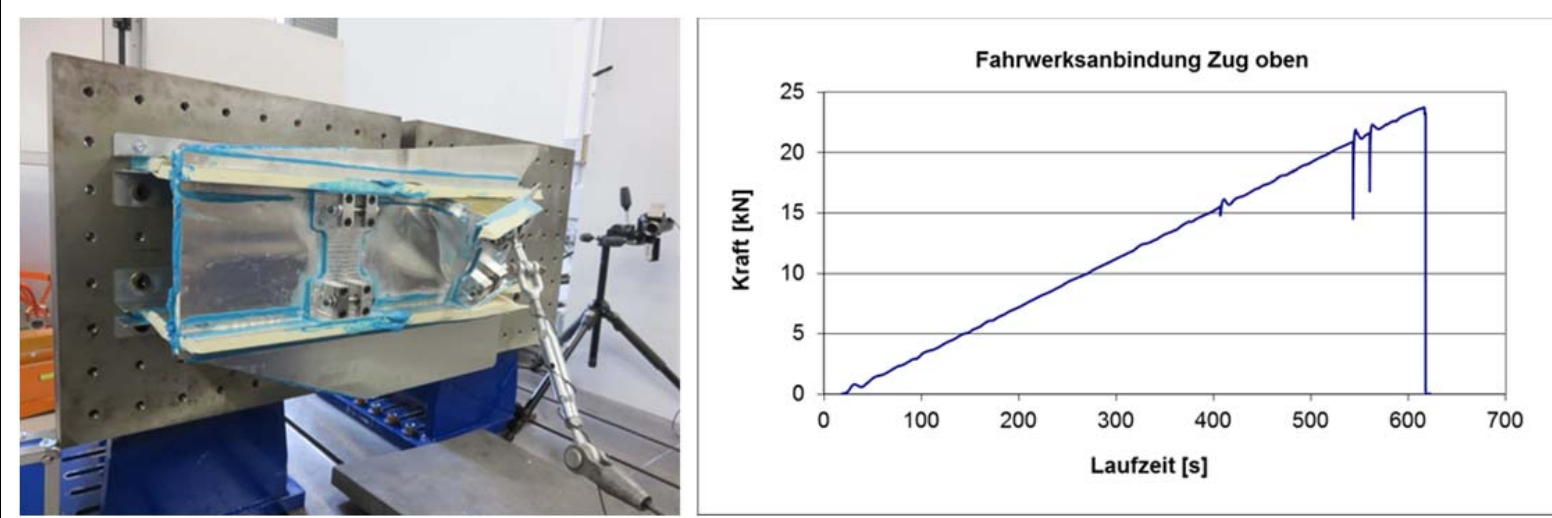

Abbildung 8: Versagen des Vorderwagens auf Zug bei circa $23 \mathrm{kN}$

\section{Weiteres Vorgehen und Ausblick}

Die Entwicklung und Validierung der Karosserie des SLRV wird von der Abteilung für Technologiemarketing des DLR gefördert. Es ist geplant, die Funktionsweise des Fahrwerks zunächst durch quasistatische Versuche zu überprüfen. Ziel ist es, im weiteren Verlauf des Projektes die Karosserie des SLRV als Demonstrator vollständig aufzubauen und auf der Crashanlage des Institutes zu testen, um so einen Abgleich zwischen Versuch und Simulation zu erhalten. Parallel dazu wird die Methodik zur Modellierung der Sandwichhalbzeuge und -werkstoffe stetig verbessert.

Weitere Arbeitsschritte im Forschungsprojekt NGC sind die Auslegung und Integration des Antriebstrangs sowie die Entwicklung des Energiesystems, unter Berücksichtigung der Klimatisierung. Auch die Untersuchung der Fahrdynamik des SLRV, sowie die Entwicklung von Türen und Klappen, Innenraum, Bedienungskonzept und Fahrerassistenzsystemen sind Teil des Projektes, welches Aktivitäten und Forschungsinfrastrukturen des DLR in den Bereichen Fahrzeugkonzepte, Fahrzeugstruktur, Antriebsstrang, Thermomanagement, Fahrzeugintelligenz und Fahrwerk, in einem Großforschungsprojekt bündelt.

Quellen

Davies, H. C., Bryant, M., \& et al. (2011). Design, development and manufacture of an aluminium honeycomb sandwich panel monocoque chassis for Formula Student competition. Journal of Automobile Engineering.

Eckstein, L., \& et al. (2011). Analyse sekundärer Gewichtseinsparpotenziale in Kraftfahrzeugen. ATZ 01/2011.

Friedrich, H. E. (2013). Alternative Antriebe und Fahrzeugkonzepte Teil I, Werkstoffe und Bauweisen. Universität Stuttgart.

Friedrich, H. E., \& Hülsebusch, D. (2009). Elektro-Fahrzeugkonzepte und Leichtbau: Anforderungen für neue Werkstoffe 1. Internationaler eCarTec Kongress für individuelle Elektromobilität, München.

Kriescher, M., \& et al. (2010). Metal-hybrid structures for an improved crash behaviour of car body structures. Materialien im Karosseriebau. 
Stapleton, S., \& Adams, D.O. (2009). Core Design for Energy Absorption in Sandwich Composites. Journal of Composite Materials 43.

Steinle, P., \& et al. (2010). Innovative Vehicle Concept for the Integration of Alternative Power Trains. Stuttgarter Symposium. 